\title{
TRIANGLES IN ARRANGEMENTS OF LINES. II
}

\section{GEORGE PURDY}

\begin{abstract}
We show that given $n$ lines in the real projective plane, no $n-1$ of which are concurrent, the number $p_{3}$ of triangular regions formed is at most $\frac{2}{5} n(n-1)$, equality being possible.

We also show that if $n>6$ then $p_{3}<\frac{7}{18} n(n-1)+\frac{1}{3}$. Grünbaum has conjectured $p_{3}<\frac{1}{3} n(n-1)$.
\end{abstract}

1. Introduction. Let $A$ be an arrangement of $n$ lines in the real projective plane $\mathbf{P}_{2}$, and let $p_{k}$ denote the number of $k$-sided regions determined by the lines. It is assumed that $A$ is not a pencil. In [1] Grünbaum raises the question of finding an upper bound for $p_{3}$ and conjectures that

$$
p_{3} \leqslant\left[\frac{1}{3} n(2[n / 2]-1)\right]<\frac{1}{3} n(n-1)
$$

for $n \geqslant 16$, and gives R. J. Canham's proof of this when $A$ is simple (no three lines concurrent). The first nontrivial results on the general problem were found by $T$. $O$. Strommer [3] who proved among other things that

$$
p_{3} \leqslant \frac{1}{3} n(n-1)+2+\frac{1}{3} \sum_{k>4}(k-6) p_{k} \text {. }
$$

In [2] we extended these results and obtained some inequalities that implied $p_{3}<\frac{5}{12} n(n-1)$. We now extend these results further, proving two results.

THEOREM 1. $p_{3} \leqslant \frac{2}{5} n(n-1)$ if $A$ is not a near pencil ( $n-1$ lines concurrent), and the result is best possible since equality occurs when $n=6$.

THEOREM 2. $p_{3} \leqslant \frac{7}{18} n(n-1)+\frac{1}{3}$ if $A$ is not a near pencil.

Remark. If $A$ is a near pencil, then $p_{3}=2 n-2$.

\section{The proofs.}

Proof of Theorem 1. Let $\boldsymbol{A}$ be an arrangement of $n$ lines in $\mathbf{P}_{2}$, not a pencil or near pencil. Let $t_{k}$ be the number of points incident with precisely $k$ lines. For a fixed line $l$ of the arrangement, let $t_{k}(l)$ denote the number of points on $l$ incident with precisely $k$ lines, including $l$. Let us call a point incident with precisely two lines a simple point, and the other points multiple points. Let $P_{1}, \ldots, P_{m}$ be the multiple points, in order, on the line $l$, with the understanding that $m=0$ if there are no multiple points on $l$. We let $I_{i}=\left(P_{i}, P_{i+1}\right)$ called the $i$ th block be the set of simple points on $l$ between $P_{i}$ and $P_{i+1}$ on $l$, with the understanding that

Received by the editors April 2, 1979.

AMS (MOS) subject classifications (1970). Primary 52A40; Secondary 05A20. 
$\left(P_{m}, P_{m+1}\right)=\left(P_{m}, P_{1}\right)$. In the event that $m=1$ we have the single block $\left(P_{1}, P_{1}\right)$. We must therefore allow for the possibility that the endpoints of $I_{i}=\left(P_{i}, P_{i+1}\right)$ are the same point. If $m=0$ there are no blocks. Each block $I_{i}$ determines $\left|I_{i}\right|+1$ line segments $\overline{P_{i} Q_{i}}, \overline{Q_{1} Q_{2}}, \ldots, \overline{Q_{k-1} Q_{k}}, \overline{Q_{k} P_{i+1}}$, where $I_{i}=\left\{Q_{1}, \ldots, Q_{k}\right\}$, where $|S|$ denotes the cardinality of the set $S$. If $I_{i}=\varnothing$, then there is only the line segment $\overline{P_{i} P_{i+1}}$. Let $1 \leqslant j \leqslant k$. The line segment $\overline{Q_{j} Q_{j+1}}$ is the side of at most one triangle, for suppose $\overline{Q_{j} Q_{j+1}}$ is incident with two triangles. Then the removal of $l$ would result in a digon (a two-sided polygon), and digons only occur in pencils, contradicting the assumption that $A$ is not a near pencil. Thus, if $p_{3}(l)$ denotes the number of triangles having a side lying on $l$, then the contribution of $I_{i}$ to $p_{3}(l)$ is at most $k+3=\left|I_{i}\right|+3$. If in fact the contribution is less and if $I_{i} \neq \varnothing$, then we say that $I_{i}$ is a deficient block. Let $D^{l}$ denote the number of deficient blocks on $l$ and $E^{l}$ the number of empty blocks on $l$. If $I_{i}=\varnothing$, then the contribution to $p_{3}(l)$ is only $\left|I_{i}\right|+2=2$. Hence, if $m \geqslant 1$,

$$
\begin{aligned}
p_{3}(l) & \leqslant \sum_{i}\left\{\left|I_{i}\right|+3\right\}-D^{l}-E^{l} \\
& =t_{2}(l)+3 \sum_{k>3} t_{k}(l)-D^{l}-E^{l} .
\end{aligned}
$$

If $m=0$,

$$
p_{3}(l) \leqslant t_{2}(l)=t_{2}(l)+3 \sum_{k>3} t_{k}(l)-D^{l}-E^{l}
$$

and so, in any case,

$$
p_{3}(l) \leqslant t_{2}(l)+3 \sum_{k>3} t_{k}(l)-D^{l}-E^{l} .
$$

If we let $E$ and $D$ denote respectively the number of empty and deficient blocks on all lines, then taking into account that triangles get counted three times and points on $k$ lines get counted $k$ times, we obtain on summation over $l$,

$$
3 p_{3}<2 t_{2}+3 \sum_{k>3} k t_{k}-E-D
$$

We need to consider so-called $F$-blocks. Let $\left|I_{i}\right|=2, I_{i}=\left\{Q_{1}, Q_{2}\right\}$, and suppose that $I_{i}$ is not deficient. Then we have the situation depicted in Figure 1, with $\left|I_{i}\right|+3=5$ triangles numbered from one to five. We call such a block a type $F$-block and it forces the existence of a point $P$ incident with at least four lines. Let us give the segments $\overline{Q_{1} P}$ and $\overline{Q_{2} P}$ weight one to indicate that they cannot occur in any other block, and give $\overline{P_{i} P}$ and $\overline{P_{i+1} P}$ weight $\frac{1}{2}$ to indicate that they can each occur in at most two blocks (or possibly $P_{i}=P_{i+1}$ ). The sum of these weights is $3 \Sigma_{l} F^{l}=3 F$, where $F^{l}$ is the number of $F$-blocks on $l$, and $F$ is the total number of $F$-blocks. The sum of these weights cannot exceed $\Sigma_{k>4} 2 k t_{k}$, and so we have

$$
\frac{4}{3} \sum_{k>4} k t_{k} \geqslant 2 F \text {. }
$$

Let us now look at a typical block $I_{i}$ which is not an empty block, a deficient block or an $F$-block. $I_{i}=\left\{Q_{1}, \ldots, Q_{k}\right\}$. See Figure 2. The triangles are marked with the symbol $\Delta$. The points $P$ and $P^{\prime}$ are each incident with three or more lines, 


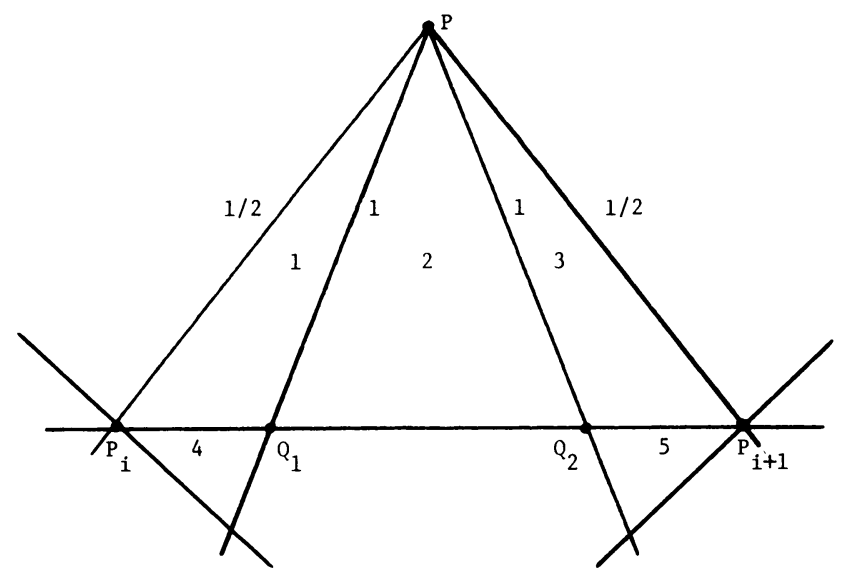

FIGURE 1

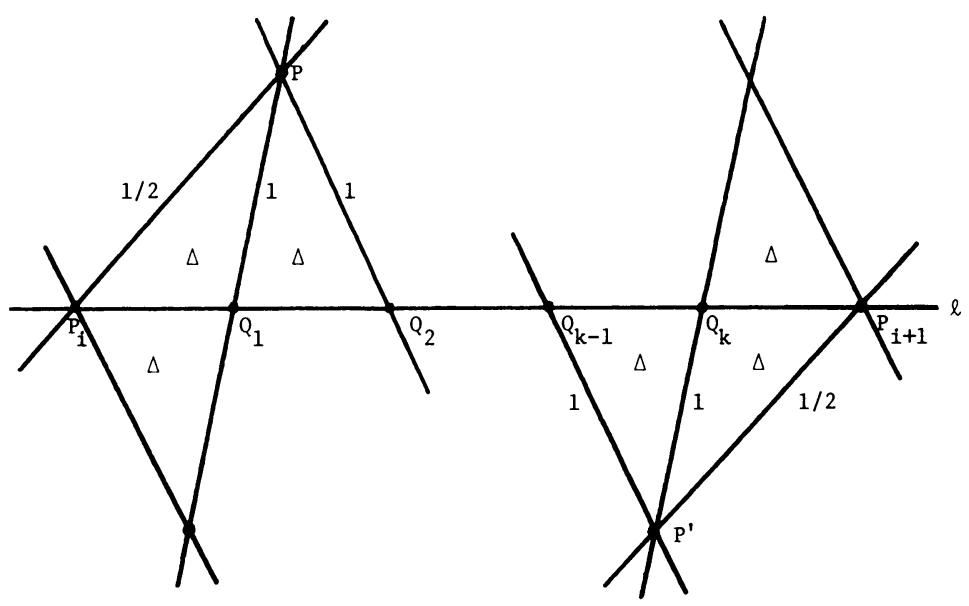

FIGURE 2

and we have given $\overline{P_{i} P}$ and $P_{i+1} P^{\prime}$ weight $\frac{1}{2}$ and we have given $\overline{Q_{1} P}, \overline{Q_{2} P}, \overline{Q_{k-1} P^{\prime}}$ and $\overline{Q_{k} P^{\prime}}$ weight one, and block $I_{i}$ has total weight 5 in the figure drawn. In fact, $P$ and $P^{\prime}$ could lie on the same side of $l$ and might even coincide. We claim that in all cases the total weight will be at least 4 . In Figure 3 we see how 4 can occur. If $P_{i}$ and $P_{i+1}$ coincide it is to be understood that each occurrence of $\overline{P_{i} P}$ in Figure 2 or 3 gets weight $\frac{1}{2}$, so that the single edge gets, in fact, a weight of one.

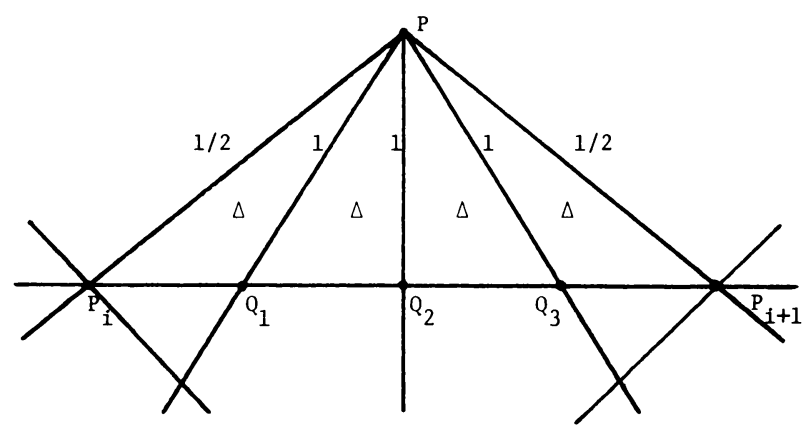

FIGURE 3 
The total weight from all of these blocks cannot exceed $\Sigma_{k>3} 2 k t_{k}$, and the number of these blocks on the line $l$ is

$$
\sum_{k>3} t_{k}(l)-F^{l}-E^{l}-D^{l}
$$

Hence

$$
\begin{aligned}
\sum_{k>3} 2 k t_{k} & \geqslant 4 \sum_{l}\left\{\sum_{k>3} t_{k}(l)-F^{l}-E^{l}-D^{l}\right\} \\
& =4\left\{\sum_{k>3} k t_{k}-F-E-D\right\},
\end{aligned}
$$

and we have

$$
2 E+2 D \geqslant \sum_{k>3} k t_{k}-2 F
$$

and adding inequality (2), we obtain

$$
2 E+2 D \geqslant 3 t_{3}-\frac{1}{3} \sum_{k>4} k t_{k}
$$

Combining with (1), we get

$$
3 p_{3} \leqslant 2 t_{2}+\frac{15}{2} t_{3}+\frac{19}{6} \sum_{k>4} k t_{k}
$$

Let $p_{k}$ be the number of $k$-gons determined by the region. Counting all of the edges, we see that $\Sigma k p_{k}=2 \sum k t_{k}$, which we rewrite as

$$
3 p_{3} \leqslant 4 t_{2}+6 t_{3}+2 \sum_{k>4} k t_{k}-\sum_{k>4} k p_{k} .
$$

Adding $\frac{4}{5}$ of (3) to $\frac{1}{5}$ of (4) we obtain

$$
\begin{aligned}
3 p_{3} & \leqslant \frac{12}{5} t_{2}+\frac{36}{5} t_{3}+\frac{44}{15} \sum_{k>4} k t_{k} \\
& \leqslant \frac{12}{5}\left\{t_{2}+3 t_{3}+\sum_{k>4}\left(\begin{array}{l}
k \\
2
\end{array}\right) t_{k}\right\}=\frac{6}{5} n(n-1),
\end{aligned}
$$

and $p_{3}<\frac{2}{5} n(n-1)$. To see that equality can occur for $n=6$, see Figure 4 , and Theorem 1 is proved.

Proof of Theorem 2. Thomas O. Strommer [3] proved

$$
p_{3}<\frac{1}{3} n(n-1)+4-\frac{2}{3} t_{2}+\sum_{k>4}(k-4) p_{k} \text {. }
$$

Adding this to (4) and multiplying the result by $\frac{3}{4}$ we obtain

$$
3 p_{3} \leqslant \frac{1}{4} n(n-1)+3+\frac{5}{2} t_{2}+\frac{9}{2} t_{3}+\frac{3}{2} \sum_{k>4} k t_{k}-3 \sum_{k>4} p_{k} .
$$




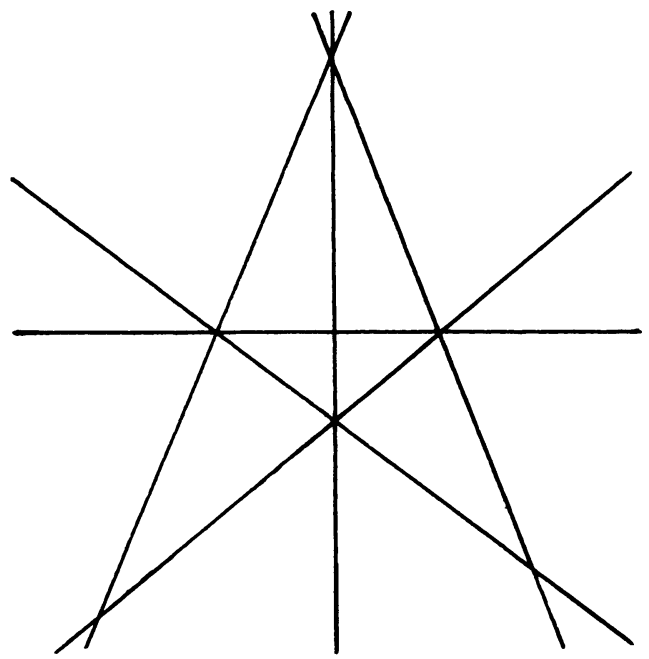

FIGURE 4. $n=6, p_{3}=12=\frac{2}{5} n(n-1)$

If we now add $\frac{1}{3}$ of (6) to $\frac{2}{3}$ of (3) we obtain

$$
\begin{aligned}
3 p_{3} & \leqslant \frac{1}{12} n(n-1)+1+\frac{13}{6} t_{2}+\frac{39}{6} t_{3}+\frac{47}{18} \sum_{k>4} k t_{k} \\
& \leqslant \frac{1}{12} n(n-1)+1+\frac{13}{6}\left\{t_{2}+3 t_{3}+\sum_{k>4}\left(\begin{array}{l}
k \\
2
\end{array}\right) t_{k}\right\} \\
& =\frac{14}{12} n(n-1)+1,
\end{aligned}
$$

and Theorem 2 follows.

REMARK. Inequality (6) gives a rather curious upper bound on $\Sigma_{k>3} p_{k}$, the total number of regions.

ACKNowledgement. We thank T. O. Strommer for helpful suggestions regarding this exposition.

\section{REFERENCES}

1. B. Grünbaum, Arrangements and spreads, CBMS Regional Conf. Ser. in Math., no. 10, Amer. Math. Soc., Providence, R. I., 1972.

2. G. Purdy, Triangles in arrangements of lines, Discrete Math. 25 (1979), 157-163.

3. T. O. Strommer, Triangles in arrangements of lines, J. Combinatorial Theory Ser. A 23 (1977), 314-320.

Department of Mathematics, Texas AdM University, College Station, Texas 77843 